Document downloaded from:

http://hdl.handle.net/10251/43342

This paper must be cited as:

Zhang, D.; Yang, Z.; Raychoudhury, V.; Chen, Z.; Lloret, J. (2013). An Energy-Efficient Routing Protocol Using Movement Trends in Vehicular Ad hoc Networks. Computer Journal. 56(8):938-946. doi:10.1093/comjnl/bxt028.

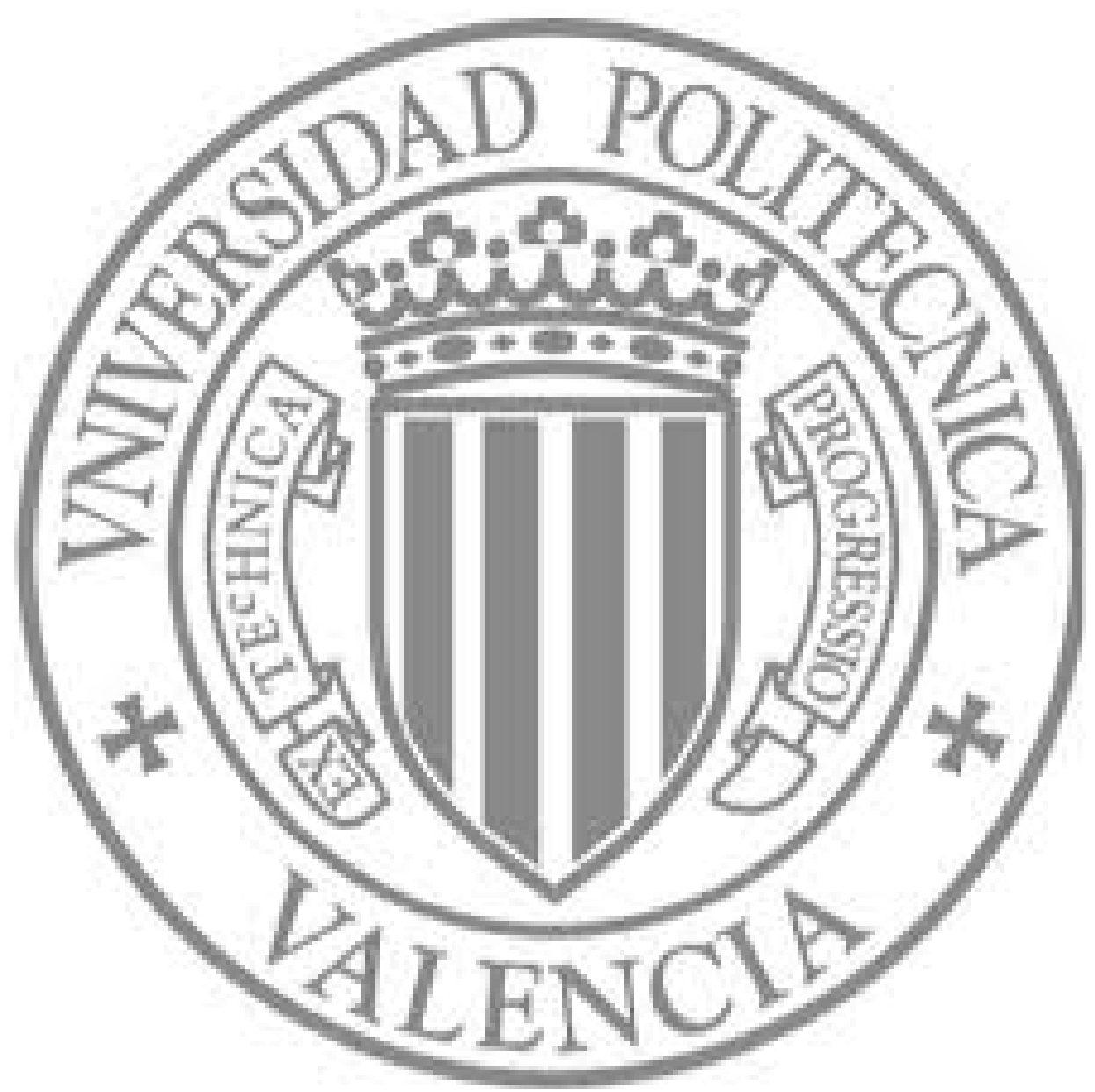

The final publication is available at

http://dx.doi.org/10.1093/comjnl/bxt028

Copyright Oxford University Press (OUP): Policy A - Oxford Open Option A 


\title{
An Energy-efficient Routing Protocol Using Movement Trends in Vehicular Ad-hoc Networks
}

\author{
DAQIANG ZHANG ${ }^{1}$, ZhiJUN YANG ${ }^{2}$, \\ VASKar RAYCHOUdhurY ${ }^{3}$, Zhe $\mathrm{CHEN}^{4}$ \\ ${ }^{1}$ School of Software Engineering \\ Tongji University, Shanghai 201804, China \\ ${ }^{2}$ School of Science and Technology \\ Middlesex University, London NW44BT, UK \\ ${ }^{3}$ Department of Electronics $\mathscr{G}$ Computer Engineering \\ Indian Institute of Technology Roorkee, Roorkee 247667, India \\ ${ }^{4}$ Department of Computer Science \\ Nanjing University of Aeronautics and Astronautics, Nanjing 210016, China \\ Email: \{dqzhang, zjyang\}@ieee.org,vaskafec@iitr.ernet.in, zhechen@nuaa.edu.cn
}

Vehicular Ad-hoc Networks (VANETs) are a killer application of Mobile Adhoc Networks (MANETs), which exchange data among vehicles and vehicles to roadside infrastructures by routing. To save energy, various routing protocols for VANETs have been proposed in recent years. However, VANETs impose challenging issues to routing. These issues consist of dynamical road topology, various road obstacles, high vehicle movement, and the fact that the vehicle movement is constrained on roads and traffic conditions. Moreover, the movement is significantly influenced by driving behaviors and vehicle categories. To this end, we incorporate them into routing and propose ERBA for VANETs - an energy-efficient routing protocol. ERBA classifies vehicles into several categories, and then leverages vehicle movement trends to make routing recommendation. It predicts the movement trends by current directions and next directions after going through the road intersections. With the vehicular category information, the driving behavior patterns, the distance between the current sections and the next intersections, ERBA propagates information among vehicles with less energy consumption. The proposed scheme is validated by real urban scenarios extracted from ShanghaiGrid project. Experimental results show that ERBA outperforms the compared routing protocols with respect to the end-end delay, the packet delivery ratio and the path duration time.

Keywords: Movement Trends, Vehicular Ad-hoc Networks, VANETs, Energy-efficient 


\section{INTRODUCTION}

Vehicular Ad-hoc Networks (VANETs) equip vehicles with wireless communication devices such that vehicles can contact each other to exchange information within a certain communication range without the underlying telecom infrastructures [1] [2] [3]. Owing to its lowcost and short-range wireless communication, VANETs are regarded as one of the most energy-efficient technologies in intelligent transportation systems. They have attracted great attention in both industry and academia. The IEEE approves IEEE 802.11 , which appends wireless access in vehicular environments to IEEE 802.11 standard [4]. Then, it further approves a higher layer standard (i.e., IEEE 1609) based on the IEEE 802.11p. Meanwhile, industrial vehicle leaders such as Bavarian Motor Works, Toyota and Volvo have launched many projects for VANETs, including Advanced Driver Assistance System [5], California PATH [6] and Sartre [7].

VANETs evolve from Mobile Ad-hoc Networks (MANETs) and Body Area Networks (BAN) [8]. In addition to the characteristics inherited from MANETs and BAN such as short-range transmission, lowcost wireless communication, self-organization and infrastructure-dependence, VANETs introduce several distinguished features. Firstly, the routes in VANETs are seriously constrained by urban environments, e.g., buildings and vehicles in the neighborhood [9]. Secondly, the topology keeps rapidly changing owing to the high-speed movement of vehicles. Thus, the topology fragmentation arises for VANETs. Thirdly, the movement of vehicles is under the constraints of road topology, traffic conditions, and traffic control signals. Finally, Global Positioning Systems (GPS) together GPS-based navigation systems enable us to dynamically collect traffic loads, the road topology, the current position, the movement trends and so on. In general, in order to foster VANET applications and facilitate drivers, the stable and reliable routing across VANETs is prerequisite.

A variety of routing protocols for MANETs, e.g., [10] [11] [12] [13] [14] [15] have been proposed during the past several years. These protocols do not take advantage of unique features of VANETs, thus being inappropriate. In recent years, there are many routing protocols put forward for VANETs, e.g., [16] [17], which are tailored to certain VANET characteristics. For instance, [18] reported a routing protocol based on the geographic topology. Several studies have uncover a regularity that the driving behaviors are closely related to sex, age, education, income, vehicle category and roads [19]. As known to all, the bus drivers drive buses in a totally different manner to that of the private car drivers. They have pre-defined routes, stations and outbound time for a period everyday. The bus drivers usually cannot drive the bus as fast as the private car drivers in most time.
Their velocity is limited.

To this end, we propose ERBA in this paper - a routing protocol based on vehicle movement trends for vehicular ad-hoc networks, which takes the driving behaviors and the vehicular categories into account. The proposed scheme is based on the fact that every type of vehicle exhibits a different kind of driving behavior. Public vehicles like buses usually run with fixed routes, stations and time, whereas private vehicles run at random. For a vehicle, to estimate the link reliability between its neighboring vehicles, ERBA makes use of its current state and motion state, the distance between its current location and next intersections, and the its neighbor vehicles. By picking out the reliable links, ERBA ensures the reliable and energy-efficient routing in VANETs. As a vehicle moves along a road section before the intersection, ERBA can estimate the duration of the current motion state. Note that ERBA finds that the driving behaviors and the next direction can improve routing performance. The finding is a performance-enhancement for existing routing protocols. We conduct a series of experiments over real urban scenarios extracted from ShanghaiGrid project [20]. Extensive experimental results show the superiority of the proposed scheme in terms of the end-end delay, the packet delivery ratio and the path duration time. In summary, the contributions of this paper consist of:

- This paper reports that the movement trends have significant effect on vehicle applications. It further incorporates the movement trends of vehicles into the routing in VANETs.

- This paper proposes a metric LRS (link reliable significance) to select the candidate vehicles for reliable and energy-efficient routing.

- Extensive experimental results show that the proposed scheme outperforms the baseline methods with respect to the end-end delay, the packet delivery ratio and the path duration time.

The rest of this paper is organized as follows. Section 2 overviews the related work. Section 3 introduces the proposed routing protocol. Section 4 reports the experimental results. Section 5 concludes the work.

\section{RELATED WORK}

Routing is a fundamental technique in ad-hoc networks as it enables message communication and remote control. In the last two decades, lots of routing protocols have been put forward. AODV [10], DSR [13], DSDV [14], OLSR [15], GPSR [21], and DCH [23] are several typical routing protocols. In this section, we briefly review the related work. Note that in the contexts without ambiguity, we use "vehicles" and "nodes" interchangeably.

Previous studies conclude that traditional routing 
protocols for MANETs cannot work properly owing to the instability, and poor performance [22] [24]. This instability is caused by network fragmentation, high vehicle movement and various road obstacles, as well as rapidly evolving traffic conditions. To this end, a magnitude of enhanced routing protocols have been proposed, e.g., [25]. These protocols leverage the knowledge of relative velocities among nodes to predict the lifetime of routes. According to the lifetime, they select routes in the route discovery and resume process. Thus, they avoid the link break among nodes before the vehicular nodes are far away.

Prediction-based routing is very close to our work. Previous work [26] and [27] initialized the first two prediction-based routing protocols. The former one periodically executed routing diagnosis to predict the next state network topology and presented a mechanism to compute vehicle movement velocity. Then it incorporated the position and the velocity of the nodes into the route selection. The last one employed the on-demand multicast routing protocol to identify reliable routes in the movement prediction process. This protocol dramatically improved the routing performance and enhanced the communication efficiency. [28] put forward the link availabilitybased routing protocol (LBRP), which took frequent network topology changes and fragile link failures into consideration. In order to estimate the route link availability and reliability, LBRP used the distance between adjacent nodes. [29] and [30] further improved the computation of the route link availability using the random-walk movement mode. According to the node movement direction and velocity, they dynamically estimated the link availability every certain interval between two nodes. Note that most existing predictionbased routing protocols for MANETs get inappropriate in VANETs. This is because VANETs bring many new challenging issues in urban traffic networks.

For VANETs, a prediction-based routing (PBR) was proposed with the support of the Internet connection [31]. PBR protocol incorporated the location and the velocity information of vehicles on routes to gateways into the route lifetime prediction. Then, it chose the routes based on the route lifetime. However, PBR protocol explicitly considered that the movement direction of a vehicle was known, either in the same direction or the opposite direction. This might not be held in urban transportation, where the vehicles have more choices of movement directions, particularly in cross roads and multi-lane roadways. In addition, the traffic conditions are much more complex than those in highways so that PBR did not work at all in urban transportation.

The Movement Prediction-based Routing protocol (MOPR) for VANETs enhanced the stability of the routing protocols proposed in [32] [33]. According to [26], MOPR made use of the route lifetime to choose the reliable routes for next route hops. Therefore, it
TABLE 1. statistics of the data set

\begin{tabular}{l|c}
\hline & GPS traces from ShanghaiGrid \\
\hline Time period & February - May , 2007 \\
Number of buses & $2,000+$ \\
Number of cars & $1,000+$ \\
Number of roads & $3,825+$ \\
Number of taxis & $4,000+$ \\
Average speed of taxis & $36 \mathrm{~km} / \mathrm{h}$ \\
Average speed of buses & $25.2 \mathrm{~km} / \mathrm{h}$ \\
Data acquisition devices & GPS and Sensors \\
Surveillance region & $240 \mathrm{~km}^{2}$ \\
\hline
\end{tabular}

was incorporated into several basic routing protocols OLSR, AODV and Greedy Perimeter Stateless Routing (GPSR). However, MOPR was seriously affected by the movement prediction and the route link lifetime estimation. Towards the construction and maintenance of reliable communication links among nodes, [34] presented another routing protocol - ROMSGP for intelligent transportation systems. This protocol classified vehicles into groups by associating the movement direction and the movement velocity with the route link availability.

As a matter of fact, the drivers have to continuously control the velocity according to the traffic signs, traffic lights, traffic flows, traffic policemen's instructions, and other unexpected conditions. They are also influenced by their driving behaviors and vehicle categories when they drive vehicles. For instance, the bus drivers exhibit different driving patterns from those of the private vehicle drivers. To figure out how the driving behaviors and vehicle categories affect the performance, we carry out extensive research.

In this paper, we propose ERBA - an energy-efficient routing protocol using vehicle movement trends and types for VANETs. It is capable of locating and maintaining reliable routes in real urban scenarios. ERBA allows for the current movement, the future motion, and the vehicle type of vehicles. In the following sections, we give a detailed description of the proposed scheme.

\section{ERBA: AN ENERGY-EFFICIENT ROUT- ING PROTOCOL USING VEHICLE MOVEMENT TRENDS FOR VANETS}

We validate the proposed scheme using the data set acquired from ShanghaiGrid project. This project was initiated for monitoring the public transportation vehicles, and then gradually has evolved into a service to serve users with traffic surveillance and traffic information [20]. Suppose that all vehicles run on roads, we plot the road map of Shanghai city, shown in Figure 1(a), where the rectangular area is our research area. Then, we select a small area and plot it out, given in Figure 1(b). In ShanghaiGrid project, every vehicle is equipped with GPS devices and sensors, so that it can acquire the following information - instant speed 


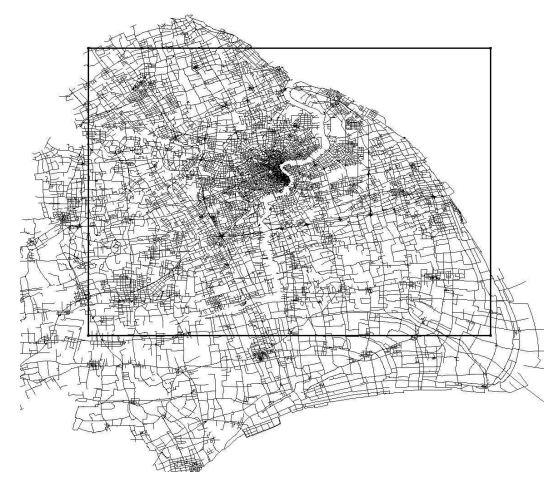

(a)

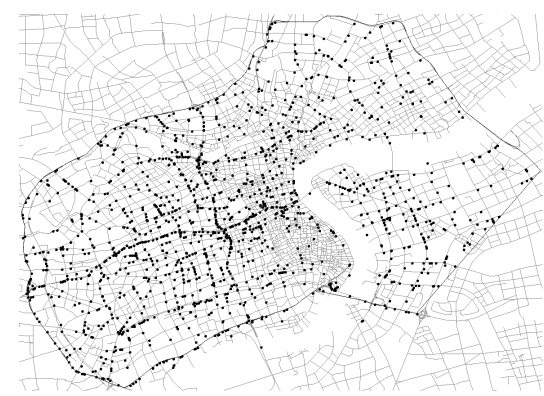

(b)

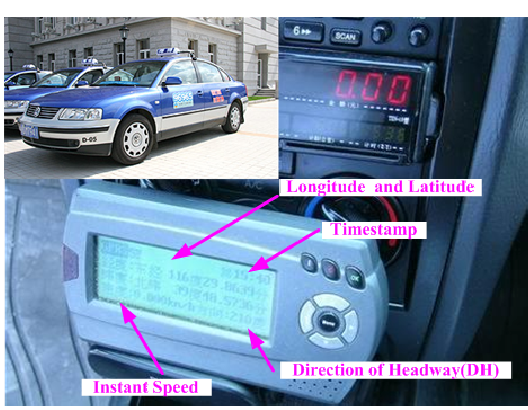

(c)

FIGURE 1. (a) The research area of Shanghai road map, where lines represent roads and black nodes refer to public buses or private cars, (b) A part of snapshots of Shanghai road map, (c) Vehicles are equipped with sensors.

TABLE 2. Notations in the design of ERBA scheme

\begin{tabular}{l|l}
\hline Notations & Explanation \\
\hline SV & the source vehicle \\
DV & the destination vehicle \\
CD & the current direction \\
ND & the next direction \\
VI & the distance between the vehicle \\
& location to the intersection \\
VT & the vehicle type \\
REQ & the route request \\
REP & the route reply \\
ERR & the route error message \\
TTL & the time-to-live of a REQ \\
LRS & the link reliable significance \\
\hline
\end{tabular}

(velocity), direction of headway, timestamp, longitude and latitude. The vehicle timely displays all this information in its onboard system, shown in Figure 1(c). Thus, the vehicles can locate itself and its neighboring intersections.

Furthermore, every vehicle maintains a neighborhood table where the position, CD, ND, VI, and the type of each neighboring vehicles are stored. The vehicle periodically updates the table by heart-beating packets. We have collected in total the information about $2,000+$ public buses, 1,000+ private cars, and 4,000+ taxies for more than three years. Note that taxi drivers share the similar drive pattern as private car drivers so that we simply consider that they are the same. Table 1 gives the statistical information of the ShanghaiGrid project. Table 2 defines the notations used in the design of the proposed scheme.

\subsection{ERBA Overview}

The ERBA protocol aims to establish reliable routes for delay-sensitive applications in urban VANETs. It is a novel prediction-based routing protocol, which predicts routes by the vehicle movement trends together with the vehicle categories. To be simple, ERBA protocol employs the routing table technique to locate routes.
ERBA allows vehicles or nodes to exchange messages through their neighbors to nodes out of their direct reach. It achieves this by discovering the routes along which messages can be exchanged. It tries to find the short and reliable routes as many as possible. It ensures that the selected routes do not contain loops. Moreover, ERBA is capable of dealing with route changes and creating new routes in case of errors.

To be specific, the proposed routing protocol works as follows.

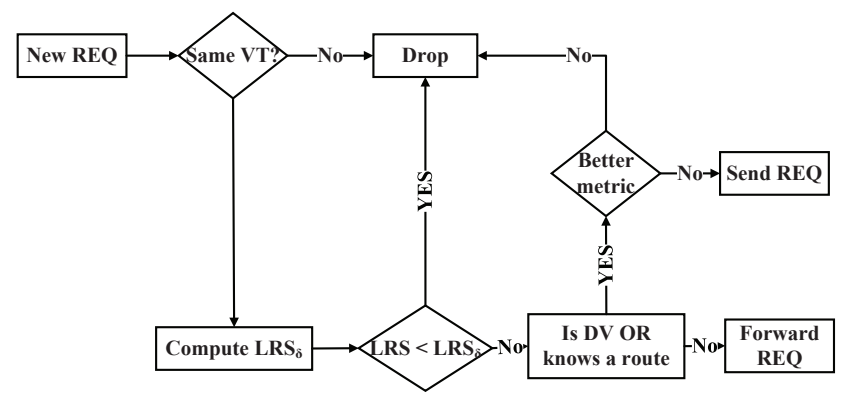

FIGURE 2. The design of ERBA scheme

- When one node (i.e., SV, the source node) needs to send a message to another node (known as DV, the destination node), it will firstly check whether the node is its neighbor. If the destination node is its neighbor, the sender and the receiver can directly communicate each other. Otherwise, the SV broadcasts a REQ (i.e., route request) message. The REQ message consists of several key bits of information: the source node, the destination node, TTL (the lifespan of the message), a sequence number (served as a unique ID), CD, ND, VI, VT and LRS (the link reliable significance of the requesting vehicle).

The sequence numbers serve as time stamps. They allow nodes to compare how "fresh" their information on other nodes is. Every time a node sends out any type of message, it increases its own 
sequence number. Each node records the sequence number of all the other nodes it communicates with. A higher sequence number signifies a fresher route. Thus, it is possible for other nodes to figure out which one has accurate information. The LRS (link reliable significance) between the requesting vehicle and the receiving vehicle is computed using $\mathrm{CD}, \mathrm{ND}, \mathrm{VI}$, and VT of the requesting vehicle.

- When a node receives a REQ message, there are two choices. Suppose the node knows a route to the destination or it is the destination, it can send a REP (route reply) message back to the source node. It will estimate the LRS of REQ packet received for a time, and then will select the REQ with the largest LRS to send REP. Otherwise, it will rebroadcast the REQ to its neighbors. The message keeps rebroadcasting until the TTL is over. Note that the receiver might drop the REQ packet in the following conditions: i) it has received the same REQ, ii) the type of requesting vehicle is different from itself, and iii) the small LRS (less than the threshold of $L R S_{\delta}$ ). Therefore, ERBA significantly avoids the REQ packet broadcasting to unreliable routing links.

Suppose the source does not receive a reply in a set amount of time, it will rebroadcast the request but this time the REQ message has a longer TTL and a new ID. All nodes use the sequence number in the REQs to ensure that they do not rebroadcast a REQ.

- The REP is sent along the vehicles which are stored in the REQ packet. Thus, the more reliable route path is set up.

- In order to improve the route stability, ERBA comes up with a preemptive mechanism to discover new routes before the route links being expired. The preemptive mechanism is discussed in Section 3.3.

\section{2. $\quad$ Routing Selection}

ERBA routing protocol collects the CD, ND, VI and $\mathrm{VT}$ of the requesting vehicle before going through the next intersections, and the distance between the vehicle and VI for reliable routes. According to the design, the REQ packet contains the CD, ND and VI of the neighbor vehicles. VI is computed through the locations of the vehicle and its neighbor intersections. Based on the $\mathrm{CD}$ and ND, ERBA predicts the movement trends. Based on the VI, it acquires the time interval before direction switch. In general, the lifespan of the routing link including the vehicles with the same CD and ND is longer than that including the vehicles with the different $\mathrm{CD}$ and ND. Moreover, the VI indicates when the vehicle keeps its current direction. The larger the VI, the longer time interval the vehicle keeps its current direction. ERBA uses a VI threshold $d_{\delta}$ to evaluate the stability of a vehicle. Only the VI is bigger than $d_{\delta}$, the corresponding vehicle can be regarded as a stable vehicle. Thus, ERBA can achieve its routing goals with less energy consumption than usual routing. Note that $d_{\delta}$ is a statistical value, which relies on specific scenes. Figure 2 illustrates the design of the routing selection.

The LRS determines the quality of routes to neighbor vehicles, which is computed as Algorithm 1. The LRS is evaluated by five ranking scale - " 5 , strongly reliable", " 4 , more reliable", "3, reliable", "2, unreliable" and "1, strongly unreliable". A routing path might be composed of many route sections. Therefore, we define the path metric related to the quality of the entire route as the minimum link metric for its path sections.

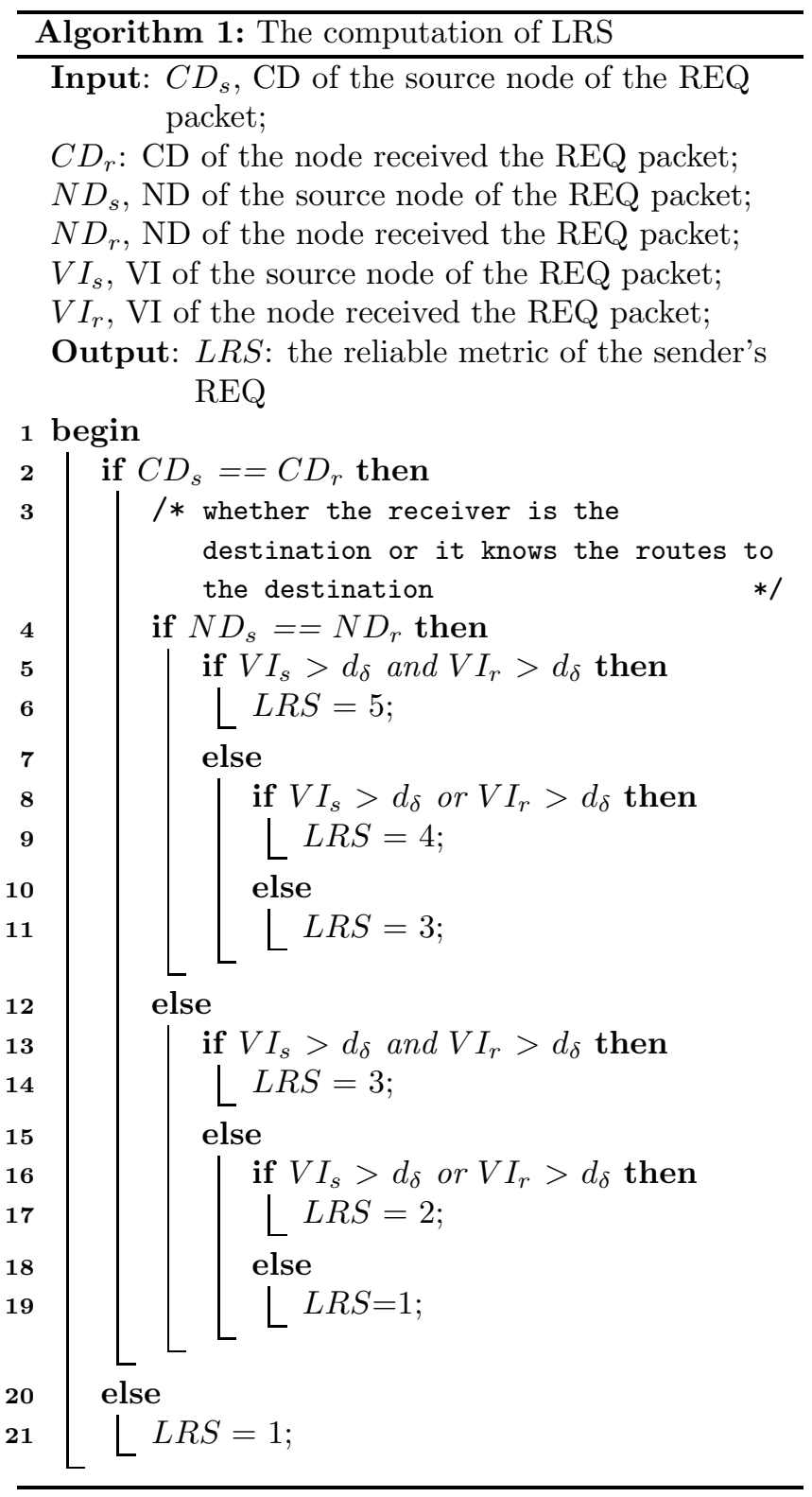

\subsection{Preemptive Route Mechanism}

ERBA puts forward a preemptive route mechanism so as to improve the stability and reliability of routing. 
When a vehicle is passing an intersection, it immediately begins to update its neighbor table by information exchange between itself and its neighbors. Then, the vehicle re-computes the values of LRS based on its newly-updated neighbor table. Once LRS is less than $L R S_{\delta}$, the vehicle will start the route discovery.

\subsection{Error Diagnosis}

ERBA leverages the ERR (route error message) to adjust routes when vehicles move around. Whenever a vehicle receives an ERR message, it looks up its routing table and removes all the routes containing the bad nodes.

ERBA just takes the following failures into consideration. One case is that a vehicle receives an ERR message that causes at least one of its routes to become invalid. If it happens, the vehicle would send out an ERR with all the new nodes which are now unreachable. The other case is that the vehicle finds that it cannot communicate with one of its neighbors. When this happens, it looks up its route table and marks them as invalid. Then, it sends out an ERR message with the neighbor and the invalid routes.

\section{EXPERIMENTS}

We carry out extensive experiments to evaluate the performance of the proposed routing protocol. We select two previous works as baseline scheme AODV [10] and ROMSGP [34] that might be regarded as canonical.

This section is organized as follows. We firstly introduce our experimental configuration, and then report experimental results.

\subsection{Experimental configuration}

We use NS 2.34 to validate the proposed scheme across the real urban scenarios that are extracted from ShanghaiGrid project. The time interval for the scenarios are from 10:00 AM to 11:00 AM and 15:00 PM to 16:00 PM. Then, we count the road distribution, road intersections, vehicles, stations, and hot roads. Recap that in ShanghaiGrid project, each bus is equipped with GPS and sensors, shown in Figure 1(c).

The movement of vehicles in real scenarios is limited by road topology, traffic signs and lights, driving patterns, etc. In our study, we focus on two kinds of vehicles - public buses and private cars. For each bus, we collect its routes, stations and outbound time from the Internet. This is because these two kinds of vehicles exhibit entirely different driving patterns. The bus drivers run the buses along the stable bus routes, stop at the bus stations, and periodically repeat the actions in most days. Whereas the car drivers drive vehicles more randomly than the bus drivers. They are both affected by road topology, traffic lights and signs, etc. In addition, the communication range
TABLE 3. Experimental settings

\begin{tabular}{l|c}
\hline Experimental parameters & Value \\
\hline Study area & $1.5 \mathrm{~km} \mathrm{X} 2 \mathrm{~km}$ \\
Study duration & $30 \mathrm{~min}$ \\
Communication range & $500 \mathrm{~m}$ \\
MAC protocol & IEEE $802.11 \mathrm{p}$ \\
Number of buses & $50-150$ \\
Number of cars & $70-300$ \\
Propagation model & Nakagami \\
Data packet size & 512 bytes \\
\hline
\end{tabular}

of vehicles is seriously limited by various obstacles, such as skyscrapers, central parks, stadiums and urban residential communities.

According to statistics, we get the probability of every kind of vehicle going straight, turning right or left at intersections. We also get the probability of vehicles turning right or left at T-junctions.

ERBA employs the IEEE 802.11p at the MAC layer for wireless communication that is dedicated to VANET applications. As for the physical layer, the proposed scheme uses Nakagami propagation model, which is realistic yet simple. Note that various propagation models lead to dramatically different performance. We omit this part as it is out of the scope of this paper. The experimental settings are shown in Table 3.

\subsection{Metrics}

In order to validate the performance, we select a series of metrics. These metrics are the packet delivery ratio, the end-end delay, and the path duration time. They are given as follows.

- The packet delivery ratio refers to a fraction of the successfully and correctly delivered data packets to all data packets. This is an elementary metric for ERBA routing protocol, as it implies whether ERBA accurately works or not.

- The end-end delay denotes the average time, which the routing protocol needs to deliver data packets from the source vehicles to the destination vehicles. This index characterizes the latency that the routing protocol might generate. Usually, given the network bandwidth, the routing protocols require low energy consumption in the case of low end-end delay.

- The path duration time defines the average duration time of the routes between two communication vehicles. This metric is used to measure the reliability of the routing protocols.

\subsection{Experiment Analysis}

4.3.1. Vehicle coverage

Fig. 3 illustrates the PDF (probability density function) as a function of the number of the vehicles. For all $\mathrm{X}$-axis values of the number of the vehicles, 


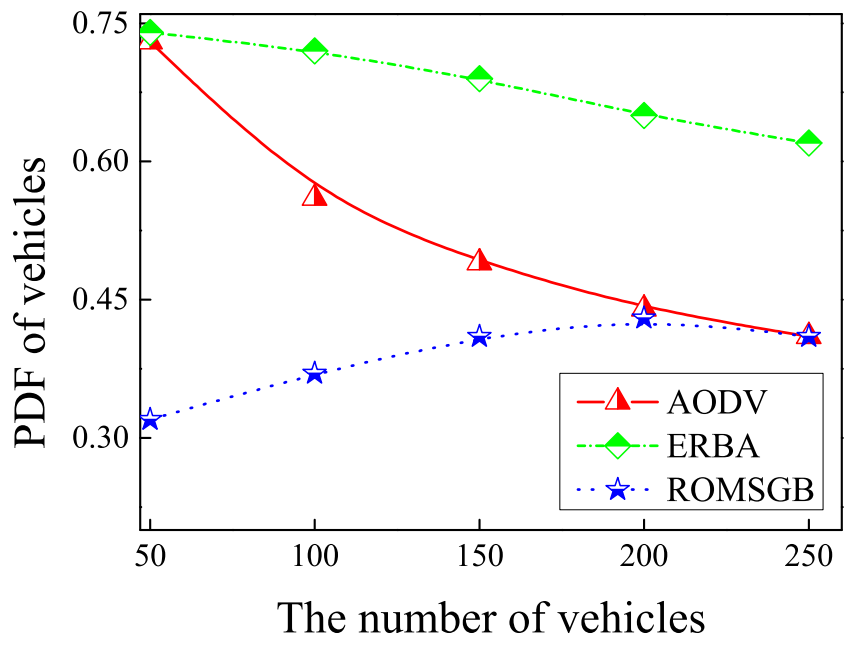

FIGURE 3. The PDF as a function of the number of vehicles

ERBA scheme achieves the highest PDF among all the routing protocols. This indicates the superiority of the proposed scheme, because ERBA covers most vehicles during the experimental process. Furthermore, ROMSGP scheme performs better than AODV scheme as the number of the vehicles increases. At the first $\mathrm{x}$ axis label (i.e., $x$ equals 50), both ERBA and AODV get a similar PDF. Whereas when the number of vehicles is 250 , the PDF of ERBA is 0.72 , almost 2 times the height of the PDF of AODV. This is because ERBA is capable of discovering a reliable route with a larger probability than AODV by movement trends prediction. ERBA ignores the REQ with low LRS. As a result, only the vehicles with high reliable links are regarded as candidates.

Meanwhile, ROMSGP also exhibits the similar trend of the vehicle coverage as ERBA, but its PDF is much lower than ERBA. This is because that ERBA chooses relay vehicles according to both the $\mathrm{CD}$ and ND. Thus, it removes the inappropriate nodes with the same CD but different ND.

\subsubsection{Throughput}

In this section, we carry out a couple of experiments to evaluate the throughput performance of the proposed routing protocol.

Table 4 illustrates the throughput of the proposed scheme. With the increase of the number of vehicles, the system throughput decreases. Among all schemes, ERBA shows the slowest downtrend. The reason is that ERBA not only responds to the first route request, but also sends a REP whenever it receives REQ packets with high LRS.

\subsubsection{End-end delay}

In this section, we conduct experiments to evaluate the end-end delay of the proposed scheme. We use the average end-end delay as the metric.
TABLE 4. The throughput of the routing protocols with varying the number of vehicles

\begin{tabular}{l|c|c|c}
\hline AODV & ROMSGP & ERBA & The number of vehicles \\
\hline 224 & 128 & 240 & 50 \\
138 & 119 & 215 & 100 \\
85 & 115 & 202 & 150 \\
78 & 98 & 190 & 200 \\
71 & 87 & 188 & 250 \\
\hline
\end{tabular}

Fig. 4 illustrates the average end-end delay as the number of vehicle increases. ROMSGP and ERBA schemes share the similar average end-end delay, much smaller than AODV. This attributes that both of them can ensure the reliable route selection, thus decreasing the number of link failure and the times of route discovery and repair.

\subsubsection{Neighbor reliable links}

In this section, we do several experiments to check how many neighbor reliable links are selected in the routing process. We select the percentage of neighbor reliable links as the metric. The smaller the percentage of neighbor reliable links, the better performance the routing protocol can achieve.

Figure 5 shows the percentage of neighbor reliable links. Apparently, ERBA has the smallest values of the percentage of neighbor reliable links for all tests among all routing protocols. Note that the differences between the values of ERBA and ROMSGP are significant. This is because ERBA scheme allows for the REQs sent by the vehicles with both the same and different directions.

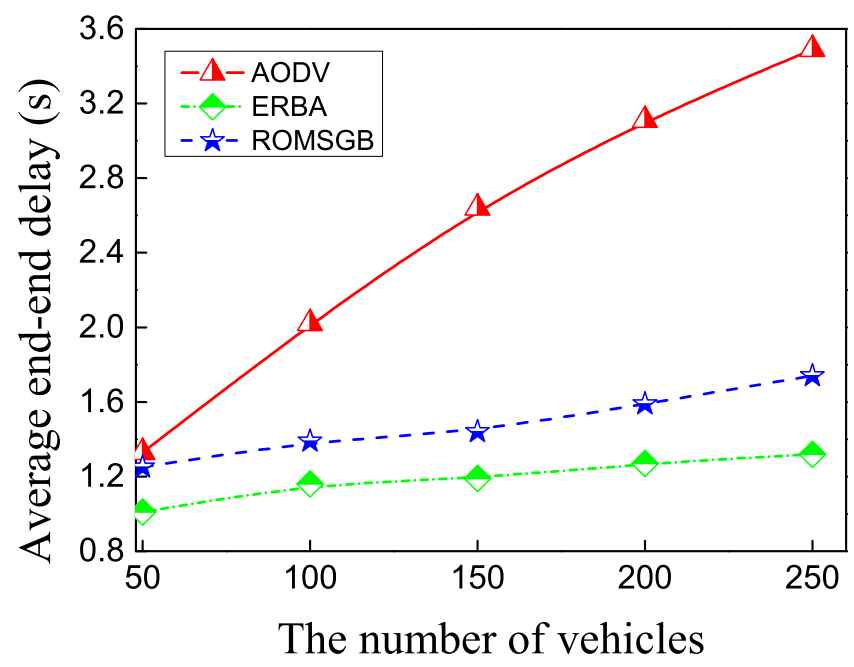

FIGURE 4. The average end-end delay of the proposed scheme as the number of vehicles increases

In summary, it is easy to infer that ERBA achieves the better performance than ROMSGP and AODV routing schemes with respect to the average end-end delay, the throughput, and the neighbor reliable links. The enhancement of ERBA attributes to the selection of the relay nodes. These nodes with high routing 
reliability are picked out by the proposed prediction mechanism.

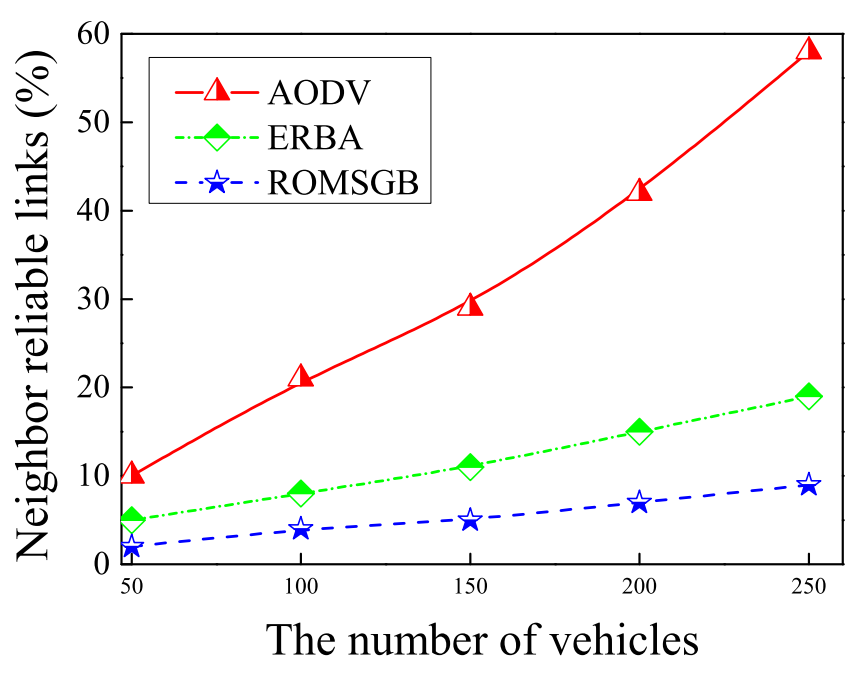

FIGURE 5. The percentage of neighbor reliable links when the number of vehicles increases

\section{CONCLUSION}

In this paper, we have proposed ERBA - an energyefficient routing protocol using movement trends in VANETs. The proposed scheme employs the driving patterns, vehicle category, and intersection information to build reliable and stable routing routes among vehicles. Extensive experimental results over real urban scenarios extracted from ShanghaiGrid project demonstrate that ERBA outperforms the compared routing schemes in VANETs.

However, ERBA could be further improved. We would like to extract more driving behavior patterns and then validate them. We will also evaluate the proposed scheme in various urban VANETs. Finally, we plan to build an independent performance-enhancement module based on the drive patterns for existing routing protocols.

\section{ACKNOWLEDGEMENTS}

This work is supported by the National Natural Science Foundation of China (Grant Nos. 61103185, 61100034, 61003247 and 61073118), the Start-up Foundation of Nanjing Normal University (Grant No. 2011119XGQ0072), and Natural Science Foundation of the Higher Education Institutions of Jiangsu Province, China (Grant No. 11KJB520009). This work is also supported by Major Program of National Natural Science Foundation of Jiangsu Province (Grant No. BK2011005).

\section{REFERENCES}

[1] Cheng, S. T., Horng, G. J., and Chou, C. L. (2011) Using cellular automata to form car society in vehicular ad-hoc networks. IEEE Transactions on Intelligent Transportation Systems, 12, 1374-1384.

[2] Yeh, L. Y., Chen, Y. C., and Huang, J. L. (2011) ABACS: an attribute-based access control system for emergency services over vehicular ad-hoc networks. IEEE Journal on Selected Areas in Communications, 29, 630-643.

[3] Zhang, D., Huang, H., Lai, C. F., Liang, X. D., Zou, Q., and Guo, M. Y. (2011) Survey on context-awareness in ubiquitous media. Multimedia Tools and Applications, 1, 1-35.

[4] IEEE P802.11p/D11.0 (2010) IEEE draft standard for amendment to standard for information technologytelecommunications and information exchange between systems-local and metropolitan networks-specific requirements-part II: wireless lan medium access control (MAC) and physical layer (PHY) specificationsamendment 6: wireless access in vehicular environments. IEEE, New York.

[5] Heddebaut, M., Rioult, J., Ghys, J., Gransart, C., and Ambellouis, S. (2005) Broadband vehicle-to-vehicle communication using an extended autonomous cruise control sensor. Measurement Science and Technology, 16, 1363-1373.

[6] Benslimane, A., Taleb, T., and Sivaraj, R. (2011) Dynamic clustering-based adaptive mobile gateway management in integrated VANET - 3G heterogeneous wireless networks. IEEE Journal on Selected Areas in Communications, 29, 559-570.

[7] Davila, A., and Nombela, M. (2011) Sartre - safe road trains for the environment reducing fuel consumption through lower aerodynamic drag coefficient. Technical paper 2011-36-0060. SAE International, USA.

[8] Chen, M., Gonzalez, S., Vasilakos, A., and Cao, H. (2011) Body area networks. ACM/Springer Mobile Networks and Applications, 16, 171-193.

[9] Zhang, D., Huang, H., Chen, M., and Liao, X. (2012) Empirical study on taxi GPS traces for vehicular adhoc networks. Proceedings of ICC, Ottawa, Ontario, Canada, 10-15 June, pp. 581-585. IEEE, New York.

[10] Perkins, C. E. and Royer, E. M. (1999) Ad-hoc on-demand distance vector routing. Proceedings of WMCSA, New Orleans, LA, USA, 25-26 February, pp. 90-100. IEEE Computer Society, New York.

[11] Wu, Y., Zhu, Y., and Li, B. (2012) Infrastructureassisted routing in vehicular networks. Proceedings of the 32nd IEEE INFOCOM, Orlando, FL, USA, 25-30 March, pp. 1485-1493, IEEE, New York.

[12] Chen, M., Leung, V. C. M., Mao, S., and Kwon, T. (2009) Receiver-oriented load-balancing and reliable routing in wireless sensor networks. Wireless Communications and Mobile Computing, 19, 405-416.

[13] Johnson, D. B., and Maltz, D. A. (1996) Dynamic source routing in ad-hoc wireless networks. In Imielinski, T., Korth, H. F., and Allen, J. (eds.), Mobile Computing, the Springer International Series in Engineering and Computer Science, 353, pp. 153-181. Springer US.

[14] Perkins, C. E., and Bhagwat, P. (1994) Highly dynamic destination-sequenced distance-vector routing (DSDV) for mobile computers. Proceedings of the International Conference on Communications Architectures, Protocols and Applications (SIGCOMM), New York, USA, 
31 August - 2 September, pp. 234-244. ACM, New York.

[15] Clausen, T., and Jacquet, P. (2003). Optimized link state routing protocol (OLSR). RFC Standard.

[16] Marc, T. M., Jens, M., Paolo, S., and Hannes, H, (2009) Vehicle-to-Vehicle communication: fair transmit power control for safety-critical information. IEEE Transactions on Vehicular Technology, 58, 3684-3703.

[17] Benslimane, A., Barghi, S., and Assi, C. (2011) An efficient routing protocol for connecting vehicular networks to the Internet. Pervasive Mob. Comput., 7, 98-113.

[18] Leontiadis, I., and Mascolo, C. (2007) GeOpps: Geographical opportunistic routing for vehicular networks. Proceedings of International Symposium on a World of Wireless, Mobile and Multimedia Networks (WoWMoM), Helsinki, Finland, 18-21 June, pp. 1-6. IEEE, New York.

[19] Shinar, D., Schechtman, E., and Compton, R. (2001) Self-reports of safe driving behaviors in relationship to sex, age, education and income in the US adult driving population. Accid. Anal. Prev., 33, 111-6.

[20] Li, M., et al. (2006) ShanghaiGrid as an information service grid. Concurr. Comput.: Pract. Exper., 18, 111-135.

[21] Karp, B., and Kung, H. T. (2000) GPSR: greedy perimeter stateless routing for wireless networks. Proceedings of MOBICOM, Boston, Massachusetts, USA, 6-11 August, pp. 243-254. ACM, New York.

[22] Lochert, C., Hartenstein, H., Tian, J., Fubler, H., Hermann, D., and Mauve, M. (2003) A routing strategy for vehicular ad-hoc networks in city environments. Proceedings of the IEEE Intelligent Vehicles Symposium, Columbus, OH, USA, 9-11 June, pp. 156-161. IEEE, New York.

[23] Wanod, K., Bhaacharya, S., Qazi, B. R., and Elmirghan, J. M. (2012) An energy efficient double cluster head routing scheme for motorway vehicular networks. Proceedings of ICC, Ottawa, Canada, 10-15 June, pp. 141-146, IEEE, New York.

[24] Naumov, V. and Gross, T. R. (2007) Connectivityaware routing (CAR) in vehicular ad-hoc networks. Proceedings of the 26th IEEE INFOCOM, Anchorage, AK, USA, 6-12 May, pp. 1919-1927, IEEE, New York.

[25] Taleb T., Sakhaee, E., Jamalipour A., Hashimoto K., Kato N., and Nemoto Y. (2007) A stable routing protocol to support its services in VANETs. IEEE Trans. Vech. Technol., 56, 3337-3347.

[26] Su, W., Lee, S. J., and Gerla, M. (2001) Mobility prediction and routing in ad hoc wireless networks. Int. J. Netw. Manag., 11, 3-30.

[27] Lee, S. J., Su, W., and Gerla, M. (2001) Wireless adhoc multicast routing with mobility prediction. Mob. Netw. Appl., 6, 351-360.

[28] Guan, Q., Yu, F., Jiang, S., and Wei, G. (2010) Prediction-based topology control and routing in cognitive radio mobile ad-hoc networks. IEEE Transactions on Vehicular Technology, 59, 4443-4452.

[29] Jiang, S. (2004) An enhanced prediction-based link availability estimation for MANETs. IEEE Transactions on Communications, 52, 183-186.
[30] Jiang, S., He, D., and Rao, J. (2005) A predictionbased link availability estimation for routing metrics in MANETs. IEEE/ACM Transactions on Networking, 13, 1302-1312.

[31] Namboodiri, V., and Gao, L. (2007) Predictionbased routing for vehicular ad-hoc networks. IEEE Transactions on Vehicular Technology, 56, 2332-2345.

[32] Menouar, H., Lenardi, M., and Filali, F. (2007) Improving proactive routing in VANETs with the MOPR movement prediction framework. Proceedings of the 7th International Conference on Intelligent Transport Systems, Los Alamitos, CA, USA, 6-8 June, pp. 1-6, Springer, Berlin.

[33] Menouar, H., Lenardi, M., and Filali, F. (2007) Movement prediction-based routing (MOPR) concept for position-based routing in vehicular networks. Proceedings of the 66th Vehicular Technology Conference, Fall, Baltimore, USA, 30 September - 3 October, pp. 21012105, Springer, Berlin.

[34] Sakhaee E., Jamalipour A., Hashimoto K., and Kato N. (2007) A stable routing protocol to support its services in VANET networks. IEEE Transactions on Vehicular Technology, 56, 3337-3347.

[35] Gozalvez, J., Sepulcre, M., and Bauza, R. (2010) Impact of the radio channel modelling on the performance of VANET communication protocols. Springer Telecommunication Systems, 1, 1-19. 